\title{
VARIACIÓN RADIAL Y LONGITUDINAL DE LA DENSIDAD BÁSICA EN ÁRBOLES DE EUCALYPTUS REGNANS DE 16 AÑOS
}

\section{RADIAL AND LONGITUDINAL BASIC DENSITY VARIATION IN 16 YEARS OLD EUCALYPTUS REGNANS TREES}

\author{
Margarette Omonte ${ }^{1}$, Luis Valenzuela ${ }^{1}$
}

\begin{abstract}
RESUMEN
La variación radial y longitudinal de la densidad básica de la madera, a lo largo del fuste, fueron determinadas en árboles de una plantación de Eucalyptus regnans de 16 años, crecida en la Costa de la Provincia de Arauco, Chile. Además, se correlacionó la densidad básica promedio del fuste, con la densidad básica obtenida a distintos niveles de altura. Se muestrearon 9 árboles entre dominantes y codominantes. De cada árbol se obtuvieron rodelas cada $10 \%$ de la altura total, hasta una altura del $70 \%$. De las rodelas se extrajeron cubos de $1 \mathrm{~cm}$ de arista, de médula a corteza en las direcciones norte y sur, y en ellos se determinó la densidad básica. Se encontraron tres patrones de variación radial para la densidad básica a lo largo del fuste del árbol. En la dirección longitudinal la densidad básica disminuyó desde la base del fuste hasta la altura del 4,5\% y luego aumentó linealmente hasta el 70\% de la altura total del árbol. Las mejores alturas de muestreo para estimar la densidad promedio del fuste, fueron al 10 y al $20 \%$ de la altura total del árbol.
\end{abstract}

Palabras claves: Eucalyptus regnans, densidad básica, variación radial, variación longitudinal, altura óptima de muestreo.

\begin{abstract}
The radial and longitudinal variations in wood basic density along the stem were determined in trees from a plantation of Eucalyptus regnans (16 year old) grown at the coast of the Arauco Province, Chile. The weighted stem basic density was estimated and correlated to the basic density at different sampling heights. Nine dominant and/or codominant trees were selected. Discs were obtained each $10 \%$ of the total height until a height of $70 \%$. From the discs, small cubic samples of $1 \mathrm{~cm}$ edge from the pith to the bark and in the north and south directions were extracted, and in these small samples the basic density was determined. Three radial variation patterns for the basic density along the stem were found. In the longitudinal direction, basic density decreased from the base of the tree to $4.5 \%$ of the height, and then increased linearly until $70 \%$ of the total tree height. The best sampling heights for estimating the weigthed basic density of the stem were to 10 and $20 \%$ of the total tree height.
\end{abstract}

Keywords: Eucalyptus regnans, basic density, radial variation, longitudinal variation, optimal sampling height. 


\section{INTRODUCCION}

Eucalytus regnans F. muell, es considerada una de las especies forestales de mayor importancia económica en Australia, su país de origen (INFOR-CONAF, 1997), donde puede alcanzar alturas superiores a los $90 \mathrm{~m}$ constituyéndose así, en la especie más alta de ese lugar y en la latifoliada más alta del mundo (FAO 1981, INFOR-CORFO 1989, Clifton 1990, INFOR-CONAF 1997). En Australia los usos de su madera son principalmente: celulosa, papel, mueblería, ebanistería, chapas, mangos de herramientas, pisos, paneles y en la construcción en general (Clifton 1990).

En Chile esta especie ha sido probada en diferentes condiciones edafoclimáticas, y ha demostrado una buena adaptación en la zona centro sur, siendo recomendada para gran parte de las Regiones del BioBío y Araucanía, además del norte de la Región de los Ríos (INFOR-CONAF, 1997).

Una propiedad fundamental de conocer en cualquier especie, es la densidad básica de la madera, definida como el peso de la madera seca (anhidra), dividido por su volumen verde $(\geq 30 \%$ Contenido de Humedad) (INFOR-CONAF 1989, Zobel y van Buijtenen 1989, Zobel y Jett 1995, Downes et al. 1997). Esta propiedad es considerada como uno de los mejores parámetros de calidad, debido a que muchas propiedades físicas y mecánicas de la madera están en función de ella. Además, este parámetro es importante para los procesos de conversión mecánica (aserrío, debobinado, cepillado, etc.), conversión química (pulpa y papel) y tratamientos de la madera (secado, impregnación, aplicación de barnices y pinturas) (INFOR-CORFO 1989).

La densidad básica varía considerablemente entre especies, entre árboles y dentro de árboles individuales (INFOR-CORFO 1989, Zobel y van Buijtenen 1989, Downes et al.1997). Esta variación se debe tanto a factores propios de cada especie como a las interacciones con el medio ambiente (INFORCORFO 1989, Zobel y Van Buijtenen 1989, Zobel y Jett 1995). Las características de la madera como: tipos de células, diámetros de lúmenes y espesor de las paredes celulares, así como el contenido de extraíbles, entre otras, también inciden en la variación de esta propiedad (INFOR-CORFO 1989, Zobel y Van Buijtenen 1989, Downes et al. 1997).

En términos generales, conocer los patrones de variación de la densidad básica, es importante para definir la utilización más adecuada de la madera (Tomazello 1985), y además, para conocer el grado de homogeneidad de la materia prima que se entrega a la industria forestal maderera (Quilhó et al. 2006). Es por ello, que durante estas últimas décadas, las estrategias de muestreo no destructivo para la predicción de la densidad básica promedio del fuste, han adquirido gran relevancia (Downes et al. 1997), ya que éstas, han permitido simplificar la evaluación y comparación entre árboles, así como entre plantaciones (Igartúa et al. 2003).

Estudios en E. regnans, sobre la variación de la densidad básica, tanto en dirección radial como en altura del fuste del árbol, son escasos. Aunque existen algunos trabajos, estos son parcializados en relación con la variación radial (Frederick et al. 1982, Chafe 1986) y con la variación longitudinal de la densidad básica (Frederick et al. 1982, Raymond et al. 1998), ya que, los árboles no fueron muestreados de manera intensiva en ambas direcciones. Por ejemplo, Chafe (1986), determinó la variación radial sólo a $1.5 \mathrm{~m}$ de altura, y Frederick et al. (1982) consideraron solo tres segmentos a lo largo del radio y cuatro alturas para determinar la variación radial y longitudinal, respectivamente. Por otro lado, Raymond et al. (1998) determinan la variación longitudinal entre 5 y el $90 \%$ de la altura total del árbol, pero sin considerar la variación radial. 
De acuerdo a lo mencionado anteriormente, los objetivos de este trabajo fueron: a) determinar exhaustivamente la variación radial y longitudinal de la densidad básica, b) correlacionar la densidad básica promedio del fuste con la densidad básica a distintas alturas de muestreo y c) validar las relaciones encontradas entre la densidad básica promedio del fuste y la densidad básica a distintas alturas de muestreo.

\section{MATERIALES Y METODOS}

\section{Antecedentes del área de estudio}

El material provino de una plantación de E. regnans, de 16 años de edad, ubicada en la costa de la Provincia de Arauco ( $\left.37^{\circ} 19^{\prime} \mathrm{LS} ; 7^{\circ} 32^{\prime} \mathrm{LO}\right)$, Región del Bio Bío, Chile. El clima del lugar es templado lluvioso, con una pluviometría media anual de $1.330 \mathrm{~mm}$ y con una temperatura máxima y mínima de $23^{\circ} \mathrm{C}$ en Enero y $6^{\circ} \mathrm{C}$ en Julio, respectivamente. El suelo es de textura franco arcillosa y arcillosa en profundidad, con estructura de bloques subangulares, muy plásticos y adhesivos en húmedo, correspondiente a la serie de suelo Curanipe (CIREN-CORFO 1983).

La plantación fue establecida en el año 1992, a una densidad inicial de 1.200 plantas por hectárea. Las intervenciones silvícolas efectuadas en el rodal fueron una poda a los 6 años de edad, hasta los 6 $\mathrm{m}$ de altura; y un raleo a los 10 años. Al momento del muestreo, el rodal tenía una densidad residual aproximada de 300 árboles/ha.

\section{Obtención de muestras}

Se escogieron 9 árboles entre dominantes y codominantes, con un DAP (diámetro a la altura de pecho, tomado a $1.3 \mathrm{~m}$ sobre el nivel del suelo) mínimo y máximo de 27.2 y $53.8 \mathrm{~cm}$, respectivamente, y con alturas totales en un rango de 26.6 a $30.5 \mathrm{~m}$ sin evidencia de daño o enfermedad y sin inclinación con respecto la posición vertical.

De cada árbol se extrajeron rodelas pareadas de $5 \mathrm{~cm}$ de espesor aproximadamente, a la altura del tocón, a la altura de pecho (AP; $1.3 \mathrm{~m}$ sobre el nivel del suelo) y cada $10 \%$ de la altura total del árbol, hasta una altura del $70 \%$. Aunque los fustes fueron muestreados hasta el $90 \%$ de la altura total del árbol, se consideró solo hasta el 70\%, debido a que los diámetros de las rodelas obtenidas sobre esta altura, fueron inferiores a un diámetro libre de utilización de $10 \mathrm{~cm}$. Como se muestra en figura 1, la rodela superior fue usada para generar modelos de predicción de la densidad básica promedio del fuste, mientras que la rodela inferior, se utilizó para validar dichos modelos, así como también, para determinar los patrones radiales y longitudinales de la densidad básica.

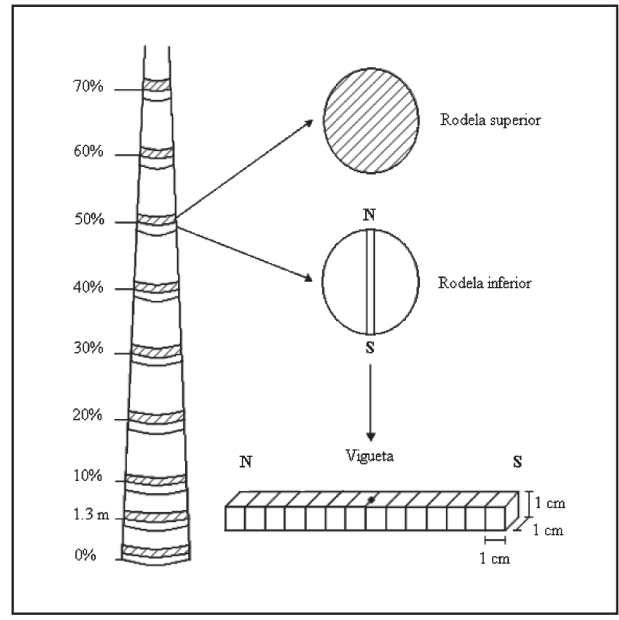

Figura 1. Posiciones de muestreo en el árbol. 
En la determinación de los patrones radiales de la densidad básica, no fue posible segregar los anillos de crecimiento, lo cual no permitió evaluar la densidad en los anillos individuales. Por esto, de las rodelas inferiores, se obtuvieron viguetas de $1 \mathrm{x} 1 \mathrm{~cm}$ en espesor y ancho, de norte a sur pasando por la médula, las cuales fueron seccionadas de médula a corteza cada $1 \mathrm{~cm}$ para ambas orientaciones, obteniéndose así, cubos de madera de $1 \mathrm{~cm}$ de arista, aproximadamente (Figura 1). Este procedimiento fue similar al utilizado por DeBell et al. (2001) en Eucalyptus saligna, pero ellos seccionaron tarugos de incremento.

\section{Determinación de la densidad básica}

Para la determinación de la densidad básica, tanto de los cubos de madera obtenidos de las rodelas inferiores como de las rodelas superiores sin corteza, se siguió las recomendaciones del método B, de la norma Americana ASTM D-2395. Esta metodología consiste básicamente, en la obtención del volumen de la madera verde por desplazamiento de agua, y posteriormente la madera verde se seca en un horno a $103 \pm 2^{\circ} \mathrm{C}$ hasta un peso constante.

Para estimar la densidad básica promedio de las rodelas inferiores, fue necesario tratar estas rodelas en dos secciones (norte y sur), debido a que en algunos casos las rodelas no fueron concéntricas con respecto a la médula. Dentro de cada sección, se ponderó la densidad básica obtenida en los cubos de madera (Figura 1), por el área de la mitad del anillo concéntrico que proyecta cada cubo con respecto a la médula (Figura 2).

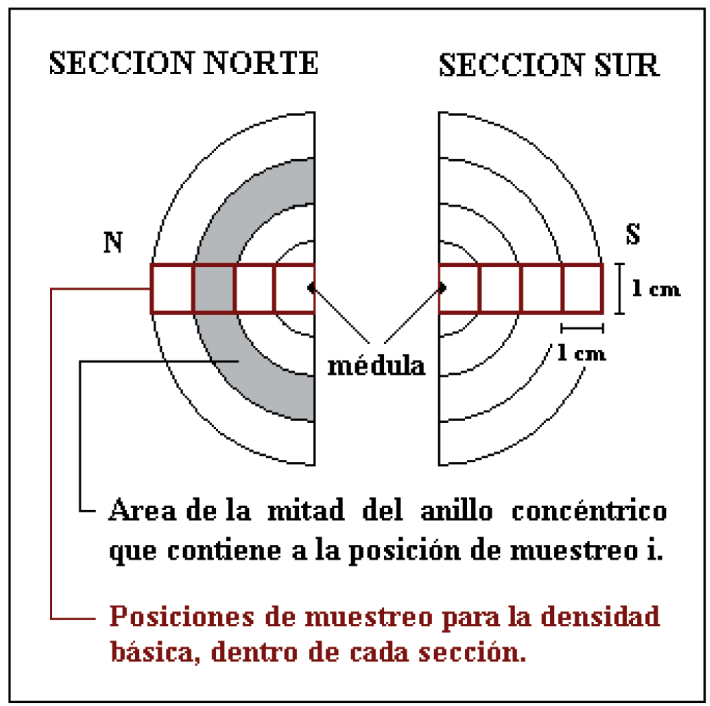

Figura 2. Secciones consideradas para estimar la densidad básica promedio de una rodela inferior $\left(\rho_{r}\right)$.

Para cada sección X de una rodela (norte o sur), se calculó la densidad básica promedio ponderada por el área $\left(\rho_{x}\right)$, a través de la siguiente ecuación:

$$
\rho_{X}=\frac{\Sigma_{i=1}^{n}\left(\rho_{X i} * A_{X i}\right)}{\sum_{i=1}^{n} A_{X i}}
$$

Donde

i: es la posición radial de muestreo de médula a corteza, cada $1 \mathrm{~cm}, \mathbf{i}=1,2,3, \ldots, \mathbf{n}$;

$\rho_{x i}:$ es la densidad básica en la posición radial de muestreo $\boldsymbol{i}\left(\mathrm{g} / \mathrm{cm}^{3}\right)$.

$A_{x i}:$ es el área de la mitad del anillo concéntrico que contiene a la posición de muestreo $\boldsymbol{i}\left(\mathrm{cm}^{2}\right)$, 
calculada de la siguiente manera:

$$
A_{X \bar{i}}=\frac{\pi}{2} *\left(r_{i}^{2}-r_{i-1}^{2}\right)
$$

Donde

$r_{i}$ : es el radio en la posición $\boldsymbol{i}$, dado por la cara tangencial más alejada de la médula $(\mathrm{cm})$

$r_{i-1}:$ es el radio de la posición $\boldsymbol{i}-1$, dado por la cara tangencial más alejada de la médula $(\mathrm{cm})$.

Finalmente, la suma de la densidad básica promedio ponderada por el área, obtenida tanto en la sección norte $\left(\rho_{N}\right)$ como en la sección sur $\left(\rho_{S}\right)$, ponderadas por el área total de las secciones norte $\left(A_{N}\right) \mathrm{y}$ $\operatorname{sur}\left(A_{S}\right)$, fue dividida por el área total de la rodela, entregando así el valor de la densidad básica promedio para una rodela inferior $\left(\rho_{I}\right)$, como sigue:

$$
\rho_{I}=\frac{\rho_{N} * A_{N}+\rho_{S} * A_{S}}{A_{N}+A_{S}}
$$

Cabe señalar, que la metodología utilizada anteriormente para el cálculo de la $\rho_{I}$, es similar a la utilizada por Jorge et al. (2000) y por Igartúa et al. (2003) en Eucalyptus globulus.

Utilizando las densidades de las rodelas superiores (Figura 1), excepto las obtenidas a la AP, se obtuvo la densidad básica promedio del fuste $\left(\rho_{F}\right)$, como sigue:

$$
\rho_{F}=\frac{\sum_{i=1}^{8}\left(\rho_{i} * V_{i}\right)}{\sum_{i=1}^{8} V_{i}}
$$

Donde

$i$ : es la troza existente entre dos alturas de muestreo consecutivas, $\mathbf{i}=1,2, \ldots ., 8$;

$\rho_{i}$ : es la densidad básica de la troza $\mathbf{i}$, calculada como el promedio de las densidades obtenidas entre dos alturas de muestreo consecutivas $\left(\mathrm{g} / \mathrm{cm}^{3}\right)$.

$V_{i}$ : es el volumen sin corteza de la troza $\mathbf{i}\left(\mathrm{m}^{3}\right)$, que fue estimado utilizando el diámetro de las rodelas superiores y la distancia entre ellas, tratando cada sección del árbol como un cono truncado, hasta $70 \%$ de la altura total del árbol, y desde esta altura hasta la altura fustal, fue tratado como un cono y ponderado por la densidad en la más alta posición de muestreo $(70 \%)$.

La metodología mencionada anteriormente, fue similar a la utilizada por otros investigadores (Raymond et al. 1998, Raymond y MacDonald 1998, Raymond y Muneri, 2001), en otras especies de Eucalyptus.

\section{Análisis de datos}

Las densidades entre las orientaciones norte y sur se trataron mediante análisis de varianza t-student, con un nivel de significancia del 95\%, en cada una de las rodelas inferiores, para los distintos niveles de altura muestreados (exceptuando la AP), en los árboles individuales.

Para determinar la variación radial de la densidad básica en cada altura, se ajustó una ecuación general de regresión simple en función de la distancia radial en porcentaje.

Mediante análisis de regresión simple, se modeló la densidad básica promedio del fuste en función de las densidades obtenidas a diferentes niveles de altura, luego estos modelos fueron validados con las densidades de las rodelas inferiores.

Para realizar el análisis de varianza y para correlacionar las distintas variables, se utilizó el Software Estadístico "Statistica v6.0". 


\section{RESULTADOS Y DISCUSION}

\section{Variación radial y en altura de la densidad básica}

Al comparar la densidad determinada en los radios Norte y Sur para las diferentes alturas, los resultados mostraron que en el $82 \%$ de las probetas (59 rodelas), la densidad promedio entre las dos orientaciones no fue estadísticamente diferente, a un nivel de confianza del $95 \%$. Por lo tanto, para determinar la variación radial de la densidad se procedió a considerar las densidades medidas en estas dos orientaciones (Figura 1).

En cada una de las alturas muestreadas, las longitudes de los radios variaron tanto entre árboles como entre las orientaciones Norte y Sur, dificultando la gráfica del patrón radial de la densidad al utilizar las distancias radiales "fijas" en centímetros. Por esta razón y porque los árboles tenían la misma edad, se decidió representar las distancias desde la médula hasta la periferia como un porcentaje del radio total (Chafe 1986), y así, recalcular la variación radial de la densidad en cada nivel de altura utilizando distancias "porcentuales".

Para recalcular la variación radial de la densidad utilizando distancias "porcentuales", se seleccionaron los datos de un árbol (183 pares) y se ajustó una ecuación general de la densidad en función de la distancia radial en porcentaje. La ecuación encontrada, fue escogida tanto por el valor del coeficiente de determinación $\left(\mathrm{R}^{2}\right)$ como por su simpleza, y ésta tuvo la siguiente forma;

$$
\rho_{D R}=\alpha+\beta * D R+\gamma * D R^{0,5}
$$

Donde

$\boldsymbol{\rho}_{\boldsymbol{D} \boldsymbol{R}}:$ es la densidad básica a una distancia radial $\boldsymbol{D} \boldsymbol{R}\left(\mathrm{g} / \mathrm{cm}^{3}\right)$;

$\boldsymbol{D R}$ : es la distancia radial a partir de la médula, como porcentaje del radio total (\%)

$\boldsymbol{\alpha}, \boldsymbol{\beta} \boldsymbol{y} \gamma$ : son los parámetros de la ecuación.

Con la ecuación (5) se ajustó la densidad para los diferentes niveles de altura en el fuste, incluyendo los datos de los nueve árboles. El resultado por nivel de altura se entrega en tabla 1, en conjunto con los parámetros estadísticos de asociación, correlación y precisión. Todos los ajustes fueron estadísticamente significantes al $1 \%$ del nivel de confianza.

Tabla 1. Parámetros de la ecuación $\rho_{D R}=\alpha+\beta * D R+\gamma * D R^{0,5}$, ajustada a diferentes niveles de altura en el fuste, y sus parámetros estadísticos de asociación, correlación y de precisión.

\begin{tabular}{|c|c|c|c|c|c|c|c|c|}
\hline \multirow{2}{*}{$\begin{array}{c}\text { Altura } \\
(\%)\end{array}$} & \multicolumn{3}{|c|}{ Parámetros } & \multirow{2}{*}{$\mathbf{R}$} & \multirow{2}{*}{$\mathbf{R}^{2}$} & \multirow{2}{*}{ EEE } & \multirow{2}{*}{$\begin{array}{c}\text { F } \\
\text { Calculado }\end{array}$} & \multirow{2}{*}{ n } \\
\hline & $\alpha$ & $\beta$ & $\gamma$ & & & & & \\
\hline 0 & 0,4279 & 0,0021 & $-0,0178$ & $0,481 * *$ & 0,231 & 0,039 & 58,3 & 391 \\
\hline 10 & 0,5276 & 0,0033 & $-0,0430$ & $0,442 * *$ & 0,195 & 0,040 & 38,1 & 318 \\
\hline 20 & 0,5535 & 0,0024 & $-0,0369$ & $0,415^{* *}$ & 0,172 & 0,048 & 30,5 & 296 \\
\hline 30 & 0,6039 & 0,0035 & $-0,0511$ & $0,460^{* *}$ & 0,211 & 0,053 & 37,0 & 279 \\
\hline 40 & 0,6090 & 0,0034 & $-0,0501$ & $0,517 * *$ & 0,267 & 0,046 & 46,0 & 256 \\
\hline 50 & 0,5843 & 0,0027 & $-0,0403$ & $0,435^{* *}$ & 0,190 & 0,046 & 25,2 & 218 \\
\hline 60 & 0,5650 & 0,0011 & $-0,0228$ & $0,528 * *$ & 0,279 & 0,036 & 31,2 & 164 \\
\hline 70 & 0,5512 & 0,0003 & $-0,0136$ & $0,542 * *$ & 0,294 & 0,036 & 24,1 & 119 \\
\hline
\end{tabular}

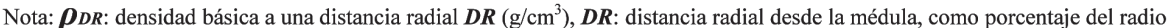
total $(\%), \boldsymbol{\alpha}, \boldsymbol{\beta}$ y $\gamma$ : parámetros de la ecuación, $\mathbf{R}$ : coeficiente de correlación, $\mathbf{R}^{2}$ : coeficiente de determinación, EEE: error estándar de estimación, F Calculado: valor de F, n: tamaño de la muestra, ${ }^{* *}$ : significante $1 \%$. 
En la figura 3 se muestra la variación radial de la densidad por nivel de altura, utilizando las ecuaciones entregadas en tabla 1, de la orientación sur de un árbol elegido al azar (DAP $=30,3 \mathrm{~cm}$ y altura total $=29,45 \mathrm{~m}$ ). Para apreciar el ahusamiento del fuste del árbol elegido, el radio a la altura de tocón fue considerado como un $100 \%$, y los otros radios de las alturas superiores, proporcionales a éste.

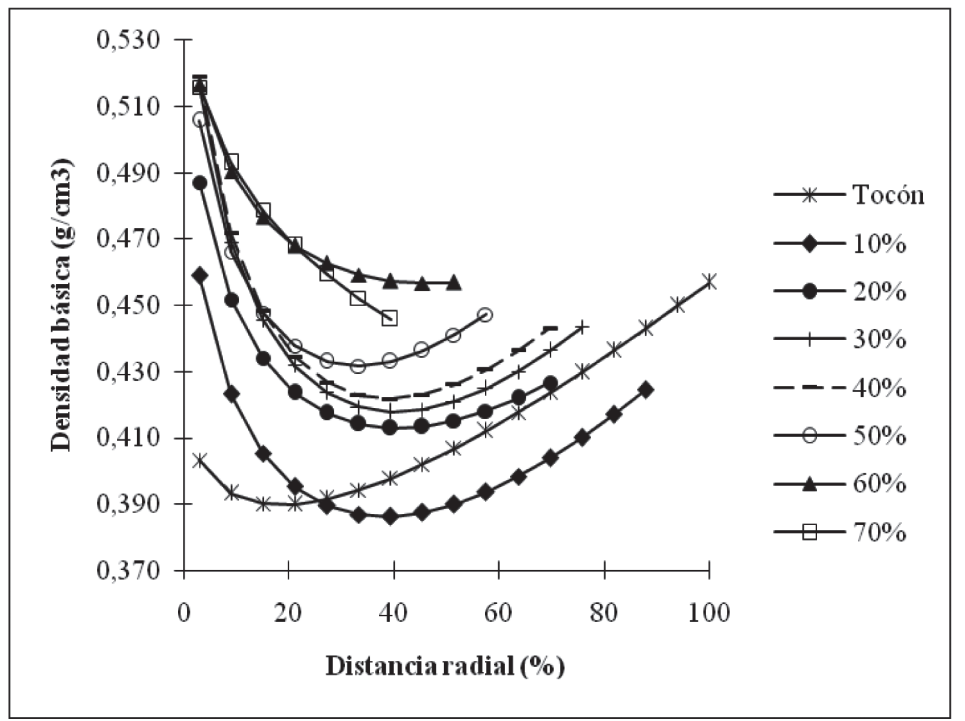

Figura 3. Variación radial de la densidad básica para cada nivel de altura muestreada en el fuste de un árbol.

De acuerdo al procedimiento anterior, se encontraron tres patrones en la variación radial de la densidad básica (Figura 3). El primero se presentó en la altura basal ( $0 \%$ de la altura total del árbol), donde la densidad disminuyó levemente, desde la médula $\left(0.403 \mathrm{~g} / \mathrm{cm}^{3}\right)$ hasta aproximadamente el $15 \%$ del radio $\left(0.390 \mathrm{~g} / \mathrm{cm}^{3}\right)$, para luego aumentar fuertemente hacia la periferia $\left(0.457 \mathrm{~g} / \mathrm{cm}^{3}\right)$. El segundo patrón fue encontrado entre el 10 y el $50 \%$ de la altura total del árbol, donde la densidad tuvo una fuerte caída desde la médula $\left(0.497 \mathrm{~g} / \mathrm{cm}^{3}\right)$ hasta aproximadamente el $40-60 \%$ del radio $\left(0.414 \mathrm{~g} / \mathrm{cm}^{3}\right)$, incrementando luego levemente hacia la periferia $\left(0.436 \mathrm{~g} / \mathrm{cm}^{3}\right)$. Finalmente, el tercer patrón radial se presenta en el 60 y $70 \%$ de la altura del árbol, donde la densidad presentó una fuerte caída de médula a corteza (de 0.516 a $0.452 \mathrm{~g} / \mathrm{cm}^{3}$ aproximadamente). Refrendando estos resultados, en las figuras $4 \mathrm{a}$, b y c, se muestran los mismos patrones descritos anteriormente, para el 60 y $70 \%$ de la altura total del árbol, para las alturas 40 y $50 \%$ y para la altura de tocón respectivamente, pero, con los datos reales en centímetros, para el mismo árbol y orientación que se muestra en la figura 3 . Al comparar de forma visual los valores de densidad entre las figuras 3 y 4, estos difieren, la razón es porque la figura 3 fue graficada con los modelos presentados en la tabla 1, y la figura 4 fue graficada con datos reales.

Por otro lado, la variación radial de la densidad, disminuyendo inicialmente desde la médula para luego incrementar hacia la periferia, ha sido reportado anteriormente en $E$. regnans a la altura de 1.5 m (Chafe 1986), en E. saligna a la AP (DeBell et al. 2001) y en Eucalyptus nitens a diferentes alturas del fuste (Lausberg et al. 1995), lo que en nuestro estudio se presentó entre el 0 y $50 \%$ de la altura total del árbol. Sin embargo, otros autores que han utilizado un menor número de segmentos radiales, para determinar el comportamiento radial de la densidad, sólo han descrito un aumento de ésta, desde médula a corteza en Eucalyptus grandis (Ferreira 1972, Tomazello 1985), E. regnans (Frederick et al. 1982), E. saligna (Tomazello 1985), E. globulus (Igartúa et al. 2000, 2002; McKinley et al. 2002) y Eucalyptus maidenii (McKinley et al. 2002). 

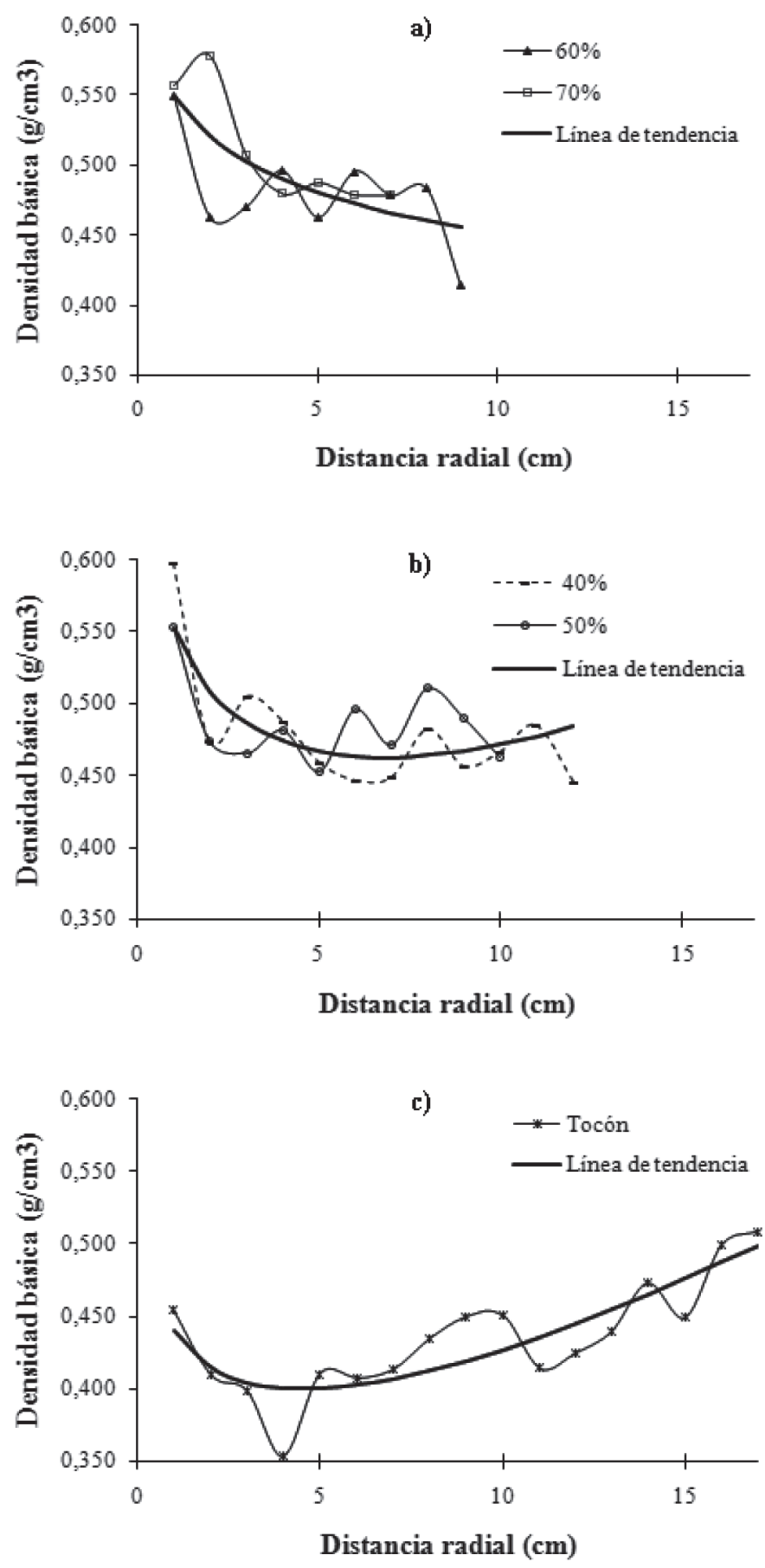

Figura 4. Variación radial de la densidad básica para el 60 y 70\% de la altura total del árbol (a), para las alturas 40 y $50 \%$ (b) y para la altura de tocón (c), con los datos reales en centímetros, para el mismo árbol y orientación que se muestra en figura 3.

La línea de tendencia es de la forma: $\rho_{D R}=\alpha+\beta * D R+\gamma * D R^{0,5}$. 
En relación al comportamiento en altura de la densidad, ésta aumentó desde la base del fuste hasta el $70 \%$ de la altura total del árbol, como se puede observar en la figura 3. Pero, un comportamiento más notorio se muestra en la figura 5, la que fue construida con la densidad promedio de los 9 árboles, obtenida para cada nivel de altura (específicamente los valores de $\rho_{I}$ ).

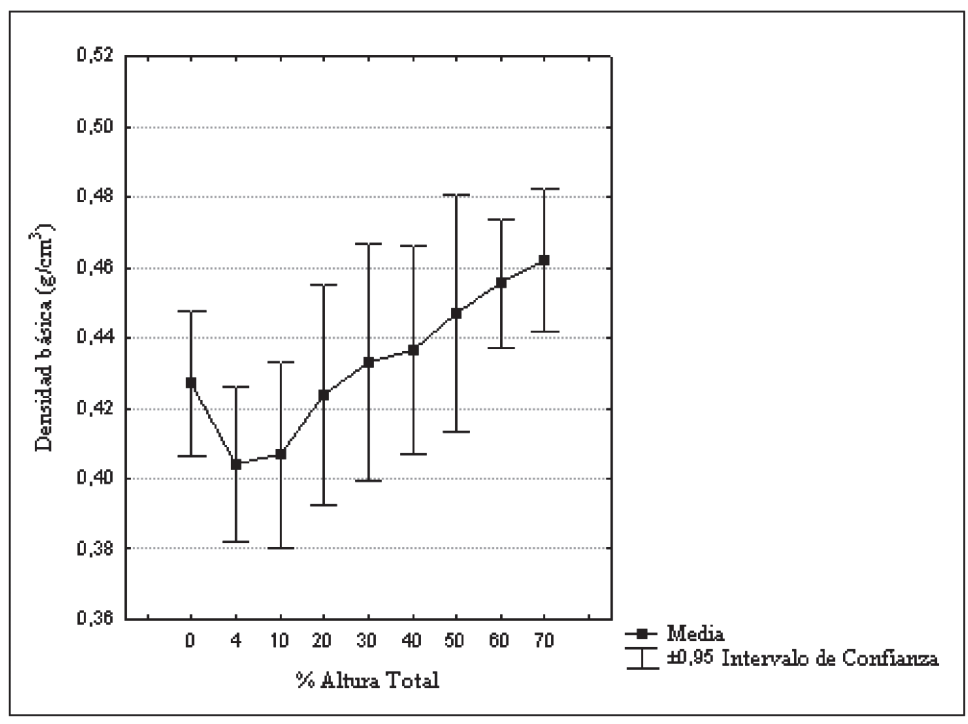

Figura 5. Variación en altura de la densidad básica, hasta el 70\% de la altura total del árbol.

De la figura 5 se desprende que la densidad disminuyó desde la base del fuste $\left(0.427 \mathrm{~g} / \mathrm{cm}^{3}\right)$ hasta aproximadamente el $4.5 \%$ de la altura total (AP promedio de los 9 árboles), registrándose en este nivel la densidad mínima $\left(0.404 \mathrm{~g} / \mathrm{cm}^{3}\right)$, y mostrando luego un aumento lineal hasta el $70 \%$ de la altura del árbol $\left(0.462 \mathrm{~g} / \mathrm{cm}^{3}\right)$. Este mismo comportamiento fue reportado por Frederick et al. (1982) en E. regnans, así como también, Lausberg et al. (1995), Raymond y MacDonald (1998), Evans et al. (2000) y Raymond y Muneri (2001) en E. nitens; y Raymond y MacDonald (1998), Raymond y Muneri (2001) y Monteoliva et al. (2002) en E. globulus, donde la densidad mínima ocurre en la primera altura de muestreo arriba de la base del árbol o altura de tocón. Sin embargo, otros investigadores que han realizado un muestreo menos intensivo en la porción basal del fuste, o que no han considerado la altura de tocón dentro de su muestreo, sólo han reportado un aumento de la densidad a lo largo del fuste del árbol, por ejemplo, Ferreira (1972) en E. grandis, Raymond et al. (1998) en E. regnans, Igartúa et al. (2000; 2002) y McKinley et al. (2002) en E. globulus y McKinley et al. (2002) en E. nitens. De lo anterior, se deduce que para futuros trabajos en densidad de la madera, se debiera considerar un muestreo mas intensivo en la porción basal del fuste de los árboles (entre 0 y $10 \%$ de la altura total), y así precisar la localización del valor mínimo de la densidad.

\section{Correlaciones entre la densidad a distintas alturas de muestreo y la densidad promedio del fuste}

La densidad básica promedio del fuste, $\boldsymbol{\rho}_{F}$, en función de la densidad básica a distintas alturas de muestreo, $\boldsymbol{\rho}_{H}$, fueron obtenidas a través de regresiones lineales simples y éstas se presentan en la tabla 2. Estas correlaciones fueron altas entre el 0 y $60 \%$ de la altura total del árbol y significantes al $1 \%$ del nivel de confianza, mientras que para la altura del 70\%, la correlación no fue significante ni a 1 ni a 5\% del nivel de confianza. Correlaciones similares fueron encontradas por Raymond et al. (1998) en $E$. regnans de 9 años de edad ( $\mathrm{n}=10$ árboles), desde un 5 a un $70 \%$ de la altura total del árbol. Aunque de acuerdo a Downes et al. (1997), Raymond et al. (1998) y Raymond y Muneri (2001), existe un cierto grado de autocorrelación, ya que, para calcular la densidad promedio del fuste se utilizaron las densidades de los distintos niveles muestreados, excepto el nivel de AP que es estadísticamente independiente. Sin 
embargo, esta autocorrelación es mínima, ya que, cada una de las alturas muestreadas fue sólo uno, de los ocho puntos utilizados en el cálculo de la densidad promedio del fuste (Neter et al. 1989).

Tabla 2. Parámetros de la ecuación $\boldsymbol{\rho}_{F}=\boldsymbol{\alpha}+\boldsymbol{\beta} * \boldsymbol{\rho}_{H}$, obtenidas a diferentes niveles de altura en el fuste, y sus parámetros estadísticos de asociación, correlación y de precisión.

\begin{tabular}{|c|c|c|c|c|c|c|c|}
\hline \multirow{2}{*}{ Altura } & \multicolumn{2}{|c|}{ Parámetros } & \multirow{2}{*}{$\mathbf{R}$} & \multirow{2}{*}{$\mathbf{R}^{2}$} & \multirow{2}{*}{ EEE } & \multirow{2}{*}{ F Calculado } & \multirow{2}{*}{$\mathbf{n}$} \\
\hline & $\alpha$ & $\beta$ & & & & & \\
\hline $0 \%$ & $-0,0235$ & 1,0411 & $0,875^{* *}$ & 0,766 & 0,016 & 22,9 & 9 \\
\hline $\mathrm{AP}$ & 0,0627 & 0,8971 & $0,902 * *$ & 0,813 & 0,014 & 30,5 & 9 \\
\hline $10 \%$ & 0,0406 & 0,9581 & $0,976 * *$ & 0,952 & 0,007 & 139,0 & 9 \\
\hline $20 \%$ & 0,0945 & 0,7915 & $0,978 * *$ & 0,956 & 0,007 & 152,3 & 9 \\
\hline $30 \%$ & 0,0918 & 0,7805 & $0,955^{* *}$ & 0,913 & 0,010 & 73,4 & 9 \\
\hline $40 \%$ & 0,0480 & 0,8716 & $0,938 * *$ & 0,880 & 0,012 & 51,2 & 9 \\
\hline $50 \%$ & 0,0849 & 0,7745 & $0,946^{* *}$ & 0,895 & 0,011 & 59,7 & 9 \\
\hline $60 \%$ & $-0,0664$ & 1,0814 & $0,838 * *$ & 0,702 & 0,018 & 16,5 & 9 \\
\hline $70 \%$ & 0,0665 & 0,7851 & $0,639 \mathrm{~ns}$ & 0,408 & 0,026 & 4,8 & 9 \\
\hline
\end{tabular}

Nota: $\boldsymbol{\rho}_{\boldsymbol{F}}$ : densidad básica promedio del fuste $\left(\mathrm{g} / \mathrm{cm}^{3}\right), \boldsymbol{\rho}_{\boldsymbol{H}}$ : densidad básica a una altura $\boldsymbol{H}$ en el fuste del árbol $\left(\mathrm{g} / \mathrm{cm}^{3}\right), \boldsymbol{\alpha}$ y $\boldsymbol{\beta}$ : parámetros de la ecuación, ns: no es significante ni al 1 ni al $5 \%,{ }^{* *}, \mathbf{R}, \mathbf{R}^{2}, \mathbf{E E E}, \mathbf{F}$ Calculado, $\mathbf{n}$, Ídem Tabla 1.

Dentro de las correlaciones se destacan las encontradas al 10 y al $20 \%$ de la altura total del árbol, por los valores de sus parámetros estadísticos, tanto de asociación como de precisión, con un coeficiente de determinación igual a 0.952 y 0.956 respectivamente, lo que indica, que solo un 4.8 y un $4.4 \%$ de la variación total de la densidad promedio del fuste no es explicada por la densidad a la altura del 10 y $20 \%$, respectivamente. En relación con este resultado, Rudman et al. (1969) citado por otros autores (Raymond y MacDonald 1998, Raymond et al. 1998, Raymond y Muneri 2001), encontraron que la densidad de las muestras tomadas al 10\% de la altura total del árbol es buena estimadora de la densidad promedio del fuste, en árboles entre 27 y 34 años de edad de E. regnans. Aunque en nuestro estudio la edad del rodal fue de 16 años, los resultados son coincidentes.

En general, para la predicción de la densidad promedio del fuste se ha utilizado la densidad obtenida al nivel de la AP o cercanas a éste, debido a que es una altura de muestreo fácilmente accesible. Para ello se han usado tanto rodelas (Ferreira 1972, Frederick et al. 1982, Igartúa et al. 2003) como tarugos de incremento (Ferreira 1972, Lausberg et al. 1995, Kibblewhite et al. 2000, McKinley et al. 2002). Aunque otros autores han utilizado la densidad de muestras obtenidas a varias alturas fijas cercanas a la base del árbol, para correlacionarlas con la densidad promedio del fuste (Raymond y MacDonald 1998, Raymond y Muneri 2001), dentro de la bibliografía revisada no se encontró que los modelos de predicción fueran validados, exceptuando a Raymond et al. (1998) en E. regnans de 10 años de edad.

En nuestro trabajo, para validar los modelos presentados en tabla 2, para la estimación de la densidad promedio del fuste, en función de la densidad a distintas alturas de muestreo, se utilizaron las densidades de las viguetas extraídas desde las rodelas inferiores (Figura 1), y que fueron calculadas como el promedio aritmético de todas las densidades obtenidas en dirección norte-sur. La relación, entre las densidades promedio del fuste estimadas por el modelo y las medidas, junto a su estadística asociada, son presentadas en la tabla 3. Los resultados muestran que las correlaciones entre los valores estimados y medidos son significantes al 1\% del nivel de confianza, desde la base hasta la altura del $50 \%$ del fuste del árbol, incluyendo el nivel de la AP, y que sólo la correlación para la altura del 70\% no fue estadísticamente significativa. 
Tabla 3. Parámetros estadísticos de asociación, correlación y de precisión obtenidos en el proceso de validación, para los modelos presentados en tabla 2 .

\begin{tabular}{cccccc}
\hline Altura & $\mathbf{R}$ & $\mathbf{R}^{2}$ & $\mathbf{E E E}$ & $\mathbf{F}$ Calculado & $\mathbf{n}$ \\
\hline $0 \%$ & $0,953 * *$ & 0,908 & 0,008 & $69,3 * *$ & 9 \\
AP & $0,886 * *$ & 0,785 & 0,012 & $25,6 * *$ & 9 \\
$10 \%$ & $0,975 * *$ & 0,950 & 0,007 & $132,2 * *$ & 9 \\
$20 \%$ & $0,975 * *$ & 0,950 & 0,006 & $133,8 * *$ & 9 \\
$30 \%$ & $0,956 * *$ & 0,913 & 0,009 & $73,9 * *$ & 9 \\
$40 \%$ & $0,849 * *$ & 0,720 & 0,017 & $18,0 * *$ & 9 \\
$50 \%$ & $0,906 * *$ & 0,820 & 0,014 & $32,0 * *$ & 9 \\
$60 \%$ & $0,748 *$ & 0,560 & 0,018 & $8,9 *$ & 9 \\
$70 \%$ & $0,592 \mathbf{n s}$ & 0,350 & 0,018 & $3,8 \mathbf{n s}$ & 9 \\
\hline Nota: *: significante $5 \%, \mathbf{n s}, * *, \mathbf{R}, \mathbf{R}^{2}, \mathbf{E E E}, \mathbf{F}$ Calculado, $\mathbf{n}$, Ídem Tabla 1.
\end{tabular}

Como fue mencionado anteriormente, los modelos generados al 10 y al $20 \%$ del nivel de altura, fueron los más precisos para la estimación de la densidad promedio del fuste (Tabla 2). Estos modelos cuando fueron validados, entregaron las mejores correlaciones entre los valores estimados y medidos, $\mathrm{R}^{2}=0.950 ; \mathrm{EEE}=0.007$ y F-test $=132.2$ para el $10 \%, \mathrm{y} \mathrm{R}^{2}=0.950 ; \quad \mathrm{EEE}=0.006$ y F-test $=133.8$ para el $20 \%$, como se muestra en tabla 3 .

Raymond et al. (1998) cuando trabajaron con E. regnans, utilizaron el modelo generado al nivel de la AP para validarlo, y encontraron un $\mathrm{R}^{2}$ de 0.490 entre los valores estimados y medidos de la densidad promedio del fuste. En cambio, en este trabajo el modelo encontrado para la misma posición de muestreo, cuando fue validado se registró un $\mathrm{R}^{2}$ de 0.785 (Tabla 3). Esta diferencia, se debe probablemente a los distintos tipos de muestras y de métodos para determinar la densidad, utilizados para validar los modelos. A diferencia de éste, Raymond et al. 1998 ocupó tarugos de incremento de $12 \mathrm{~mm}$ de diámetro, de corteza a corteza, y el método de contenido de humedad máximo para la determinación de la densidad básica. 


\section{CONCLUSIONES}

El patrón de variación radial de la densidad básica varía a lo largo del fuste del árbol.

La densidad básica disminuye desde la base hasta el nivel de la AP, y luego aumenta hasta el 70\% de la altura total del árbol.

La mejores alturas de muestreo para estimar la densidad básica promedio del fuste, fueron al $10 \mathrm{y}$ al 20\% de la altura total del árbol.

Las relaciones entre las densidades promedio del fuste estimadas por los modelos y las medidas, fueron altamente significantes entre la base y el 50\% de la altura total del árbol, destacándose las relaciones encontradas a las alturas del 10 y el $20 \%\left(\mathrm{R}^{2}=0.950\right.$ en ambos casos $)$.

\section{AGRADECIMIENTOS}

Los autores hacen expreso su agradecimiento a la Empresa Regnans Ltda., por facilitar el material para el desarrollo de este estudio.

\section{BIBLIOGRAFIA}

ASTM. 1998. Standard Test Methods for Specific Gravity of Wood-Based Materials (D-2395). Annual Book of ASTM Standards. Section 4, Construction vol 0410 Wood. American society for testing and materials. Easton. 666 p.

Chafe, S.C. 1986. Radial variation of collapse, volumetric shrinkage, moisture content and density in Eucalyptus regnans F. Muell. Wood Science and Technology 20: 253-262.

CIREN-CORFO. 1983. Ortofotos VIII Región, Anexo regional. Santiago. Chile. 17 p.

Clifton, N.C. 1990. New Zealand Timbers: Exotic and Indigenous. Wright \& Carman Ltda. Upper Hutt. Nueva Zelanda. 170 p.

DeBell, D.S.; Keyes, C.R.; Gartner, B.L. 2001. Wood density of Eucalyptus saligna grown in Hawaiian plantations: effects of silvicultural practices and relation to growth rate. Australian Forestry 64 (2): 106-110.

Downes, G.M.; Hudson, I.L.; Raymond, C.A.; Dean, G.H.; Michell, A.J.; Schimleck, L.R.; Evans, R.; Muneri, A. 1997. Sampling plantation eucalypts for wood and fibre properties. CSIRO Publishing, Melbourne. Australia. 132 p.

Evans, R.; Stringer, S.; Kibblewhite, R.P. 2000. Variation of microfibril angle, density and fibre orientation in twenty-nine Eucalyptus nitens trees. Appita 53 (5): 450-457.

FAO. 1981. El Eucalipto en la repoblación. Roma. 723 p.

Ferreira, M. 1972. Variacao da densidade basica da madeira de povoamentos commerciais de Eucalyptus grandis Hill ex Maiden nas idades de 11, 12, 13, 14 e 16 años. IPEF 4: 65-89.

Frederick, D.J.; Madgwick, H.A.I; Oliver, G.R. 1982. Wood basic density and moisture content of young Eucalyptus regnans grown in New Zealand. New Zealand Journal of Forestry Science 12 (3): 494-500. 
Igartua, D.V.; Rivera, S.M.; Monterubbianesi, M.G.; Monteoliva, S.E.; Farina, S.; Carranza, S.L; Villegas, M.S. 2000. Calidad del leño en Eucalyptus globulus ssp. globulus. I. Variación de la densidad básica y la longitud de fibra en una estación del sudeste de la provincia de Buenos Aires, Argentina. Actas Congreso Iberoamericano de Investigación en Celulosa y Papel, Misiones, Argentina.

Igartua, D.V.; Monteoliva, S.E.; Monterubbianesi, M.G; Villegas, M.S. 2002. Calidad del leño en Eucalyptus globulus ssp. globulus: II. Variaciones en la densidad básica y la longitud de fibras en Lobería, Provincia de Buenos Aires, Argentina. Revista de la Facultad de Agronomía, La Plata 105 (1): 29-39.

Igartua, D.V.; Monteoliva, S.E.; Monterubbianesi, M.G.; Villegas, M.S. 2003. Basic density and fibre length at breast height of Eucalyptus globulus ssp. globulus for parameter prediction of the whole tree. IAWA Journal 24 (2): 173-184.

INFOR-CONAF. 1997. Monografía Eucalyptus regnans. Neuenschwander \& Cruz. Santiago. Chile. 102 p.

INFOR-CORFO. 1989. Eucalyptus: Principios de silvicultura y manejo. Impresos Nova Ltda. Santiago. Chile. 199 p.

Jorge, F.; Quilho, T.; Pereira, H. 2000. Variability of fibre length in wood and bark in Eucalyptus globulus. IAWA Journal 21 (1): 41-48.

Kibblewhite, R.P.; Riddell, M.J.C.; Shelbourne, C.J.A. 2000. Variation in wood, kraft fibre, and handsheet properties among 29 trees of Eucalyptus regnans, and comparison with E. nitens and $E$. fastigata. New Zealand Journal of Forestry Science 30 (3): 458-474.

Lausberg, M.J.F.; Gilchrist, K.F.; Skipwith, J.H. 1995. Wood properties of Eucalyptus nitens grown in New Zealand. New Zealand Journal of Forestry Science 25 (2): 147-163.

McKinley, R.B.; Shelbourne, C.J.A.; Low, C.B.; Penellum, B.; Kimberley, M.O. 2002. Wood properties of young Eucalyptus nitens, E. globulus and E. maidenii in Northland, New Zealand. New Zealand Journal of Forestry Science 32 (3): 334-356.

Monteoliva, S.; Nuñez, C.; Igartua, D. 2002. Densidad básica, longitud de fibras y composición química de la madera de una plantación de Eucalyptus globulus en la provincia de Buenos Aires, Argentina. Actas II Congreso Iberoamericano de Investigación en Celulosa y Papel, Campinas, Brasil.

Neter, J.; Wasserman, W.; Kutner, M.H. 1989. Applied linear regression models. Second Edition. Irwin. Boston. USA. 667 p.

Quilho, T.; Miranda, I.; Pereira, H. 2006. Within tree variation in wood fibre biometry and basic density of the urograndis eucalypt hybrid (Eucalyptus grandis $x$ E. urophylla). IAWA Journal 27 (3): 243-254.

Raymond, C.A.; Banham, P.; Macdonald, A.C. 1998. Within tree variation and genetic control of basic density, fibre length and coarseness in Eucalyptus regnans in Tasmania. Appita 51 (4): 299-305.

Raymond, C.A.; Macdonald, A.C. 1998. Where to shoot your pilodyn: within tree variation in basic density in plantation Eucalyptus globulus and E. nitens in Tasmania. New Forests 15: 205-221. 
Raymond, C.A.; Muneri, A. 2001. Nondestructive sampling of Eucalyptus globulus and E. nitens for wood properties. I. Basic density. Wood Science and Technology 35: 27-39.

Rudman, P.; Higgs, M.; Davidson, J.; Malajczuk, N. 1969. Breeding eucalypts for wood properties. Second World Consultation on Forest Tree Breeding, Washington, USA, August, 1969. FO-FTB-69-4/7. (citado por Raymond y MacDonald (1998), Raymond et al. (1998) y Raymond y Muneri (2001)).

Tomazello, M. 1985. Variacao radial da densidade básica e da estrutura anatomica da madeira do Eucalytus saligna e E. grandis. IPEF 29: 37-45.

Zobel, B.J.; Jett, J.B. 1995. Genetics of wood production. Springer-Verlag, Berlin. Germany. 337 p.

Zobel, B.J.; Van Buijtenen, J.P. 1989. Wood variation: Its causes and control. Springer-Verlag, Berlín. Germany. 363 p. 